\title{
The effect of teicoplanin coating on osteointegration of titanium screws: a biomechanical and histomorphometric study in a rabbit model
}

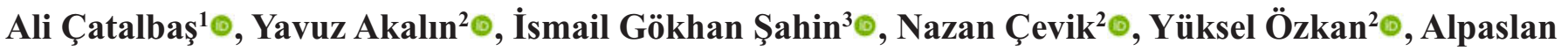 \\ Öztürk
}

${ }^{1}$ Department of Orthopaedics and Traumatology, Osmaniye State Hospital, Osmaniye, Turkey

${ }^{2}$ Department of Orthopaedics and Traumatology, University of Health Sciences, Bursa Yüksek Ihtisas Training and Research Hospital, Bursa, Turkey

${ }^{3}$ Department of Orthopaedics and Traumatology, Sultan 1. Murat State Hospital, Edirne, Turkey

\section{ABSTRACT}

Objectives: The aim of this experimental animal study was to make a biomechanical and histomorphometric evaluation of the effects of titanium screws covered with teicoplanin, which is wanted to prevent the development of infection, on osteointegration of the screw.

Methods: Twenty New Zealand white rabbits were randomly separated into 2 groups. In Group 1, 2 mini screws with teicoplanin coating were placed in the femoral condyles of the right knee and in Group 2, 2 mini screws with no coating. After 4 weeks, all the animals were sacrificed and prepared for biomechanical and histological examinations.

Results: In the pull-out test, the values of Group 1 were found to be higher and in the removal torque test, the values of Group 2 were higher. No positive correlation was found between the pull-out and removal torque tests $(\mathrm{r}=0.88)$. The bone-implant contact value was found to be similar in both groups $(p=0.132)$.

Conclusions: The results showed that titanium screws with teicoplanin coating did not interfere with osteointegration process biomechanically andhistomorphometrically by comparison with screws having no coating so that teicoplanincoating can be considered for use in orthopedic devices and joint prosthesis to prevent the development of infection.

Keywords: teicoplanin, osteointegration, histomorphometry, biomechanics, titanium

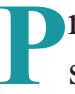
revention of implant-associated infections are essential for orthopaedic surgeons which are devastating for the patients and difficult to treat for the surgeons. Incidence has been reported to be $0.7 \%$ to 4.2 and may reach $33 \%$ in cases of high-energy trauma injuries [1-5]. Treatment is extremely difficult with very high costs and despite long-term antibiotic use, complete recovery might not be obtained. If this is the case, it is necessary to remove the orthopedic device or joint prosthesis $[1,2,5]$. In the majority, the removal of the device and prosthesis, placement of antibiotic-loaded cement spacer, long-term antibiotic treatment, and multiple debridements and revision operations cause a great increase in the cost of treatment and labor force losses $[1,6]$. In the treatment of these infections, systemic and local antibiotics are

How to cite this article: Çatalbaş A, Akalın Y, Şahin IG, Çevik N, Özkan Y, Öztürk A. The effect of teicoplanin coating on osteointegration of titanium screws: a biomechanical and histomorphometric study in a rabbit model. Eur Res J 2020;6(5):401-408. DOI: 10.18621/eurj.600539

Address for correspondence: Alpaslan Öztürk, MD., Professor, University of Health Sciences, Bursa Yüksek Ihtisas Training and Research Hospital, Department of Orthopaedics and Traumatology, 16310 Ylldırım,Bursa,Turkey.E-mail: alpaslan.ozturk@sbu.edu.tr 
applied following the removal of the device and especially joint prosthesis [1-4, 6-11]. Since there are some side effects with the use of systemic antibiotics, the application of local antibiotics has become an area of more current research $[12,13]$. In recent years, an experimental study showing the effects of teicoplanin and clindamycin coated titanium wires in the prevention of infection has been published [11]. Coating of the implant with antibiotic forms an important stage in the prevention of bacterial colonization of the implant surface starting from the moment of implantation and continuing with the expression of active substance [14]. Coating implants with antibiotics seems to be an effective method in the prevention of infections and successful results have been reported from experimental studies related to this method [8-11]. But, one might worry whether antibiotic coating interferes with osteointegration. There is a limited number of studies on the subject of the effect of the antibiotic coating of implants on osteointegration $[5,7,9-11,15]$. We hypothesized that titanium implants with teicoplanin coating did not interfere with osteointegration process biomechanically or histomorphometric. So, we decided to conduct an experimental animal study in order to see the effect of teicoplanin coating on osteointegration.

\section{METHODS}

\section{Animals and Surgery}

Approval for this experimental study was granted by the Local Ethics Committee of Animal Experiments of Uludag University(decision no: 2009-11/01, dated 15.12.2009). The number of animals was decided with power analysis and a total of 20 adults, female, healthy, New Zealand White (Oryctolaguscuniculus L) rabbits weighing with a mean of $3.10 \mathrm{~kg}$ (range, 2.85-3.36) and with a mean age of 6 months with simple randomization were separated into 2 groups and included in the study. All procedures were performed into the Experimental Animal Research Center of Uludag University Veterinary Faculty. The nutrition and care of the animals were provided by the professionals of the relevant center.

\section{Screws}

In this study, a total of 40 titanium mini-screws of 2 $\mathrm{mm}$ in diameter and $7 \mathrm{~mm}$ in length were used. The surfaces of the screws were covered with 200 mesh silica at 6 bar pressure. The process of the antibiotic coating was applied directly without any supporting system as used in the same coating procedures in literature $[1,11,12]$. Separate methanol solutions containing $16 \mathrm{mg} / \mathrm{mL}$ teicoplanin were sprayed directly onto the sanded surfaces of the titanium mini-screws. The screws were left to dry at room temperature. Following this process, they were placed in sterilization packaging and were sterilized with cobalt-60 (Co-60) gamma rays at a dose of 25.2 kilograys (kGy).

\section{Surgery}

All the operations were performed by the same surgery team in a blinded fashion. Following general anesthesia induction with ketamine $\mathrm{HCl} 35 \mathrm{mg} / \mathrm{kg}$, it was maintained with intramuscular (im) application of xylazine $\mathrm{HCl}$ and ketamine $\mathrm{HCl} 0.3 \mathrm{mg} / \mathrm{kg}$. Prophylactic antibiotic administration was started 30 min-

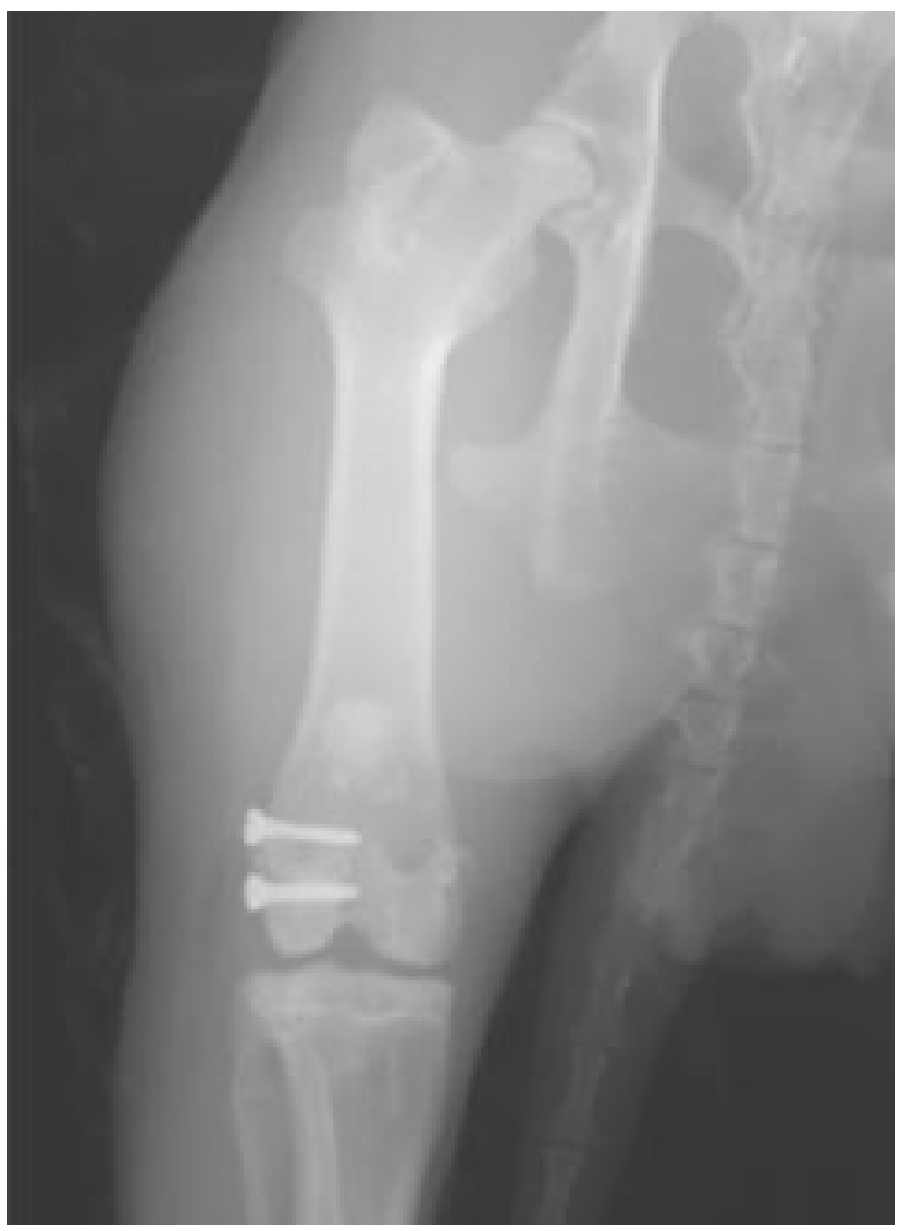

Fig. 1. Postoperative X-Ray of rabbit shows the 2 screws in the metaphyseal area of the femur. 
utes (min) preoperatively with $40 \mathrm{mg}$ /day cefazolin sodium im and was continued for 72 hours. Carprofen SC was administered as pain relief preoperatively and immediately postoperatively, then was continued for 3 days at a dose of $4 \mathrm{mg} / \mathrm{kg}$. Preoperatively, the right hind leg of each rabbit was shaved and washed with benzalkonium chloride solution, then stained with $10 \%$ iodine-povidone solution $\left(\right.$ Batticon $^{\circledR}$ ). Following the necessary draping, a $3 \mathrm{~cm}$ straight skin incision was made from the lateral side of the right patella. Skin and subcutaneous tissue were dissected, then arthrotomy was made with a lateral parapatellar cut and the femoral lateral condyle was reached. The bed for the screw was prepared in the bone with a drill bit of $1.5 \mathrm{~mm}$ in diameter and irrigation with an isotonic solution to prevent overheating. Using a screw-driver, 2 screws were placed in the metaphyseal area of each femur at a distance of $10 \mathrm{~mm}$ from each other (Fig. 1). In Group $1(\mathrm{n}=10)$ rabbits, 20 titanium mini-screws with teicoplanin coating were placed and in Group $2(\mathrm{n}=10), 20$ titanium mini-screws with no coating. The skin was sutured with $3 / 0$ monofilament nylon. The wound was closed with a sterile dressing. Postoperatively, anterior-posterior and lateral radiographs were taken to check the position of the screws. All the screws were seen to be in the lateral side of the distal femoral region, with 1 screw distal and 1 proximal. At the end of 4 weeks, all the rabbits were sacrificed by im pentobarbital $(100 \mathrm{mg} / \mathrm{kg})$ injection. The distal femurs containing the implants were removed with en bloc resection and were prepared for testing.

\section{Biomechanical evaluation and Histomorphometry}

The biomechanical evaluation was applied to a total of 14 rabbits (randomly 7 rabbits from each group), with the pull-out test applied to 14 screws and the removal torque test to 14 screws. The remaining 6 rabbits (3 from each group) were prepared for histomorphometricanalysis by cleaning the surrounding soft tissues from the right femurs and then fixing in $10 \%$ formaldehyde. For the removal torque test, the implants were resected together with the surrounding bone. For this test, 1 of the 2 implants in the femur was prepared. The removal torque test was applied to 7 implants from each group. The resected samples were wrapped in saline-soaked sponges and were stored at $-80^{\circ} \mathrm{C}$ until testing. For the test, the samples were thawed at room temperature, then fixed in standard testing equipment. After fixing the part with the implant into the testing device, removal force was applied manually, slowly and in a gradually increasing manner in an anti-clockwise direction with the digital torquemeter device probe. The process was halted when the bone with the implant started to turn within its bed. The highest torque value obtained at the moment of breaking was recorded on the digital screen as Newton/centimeter (N/cm) (Fig. 2). For the pullout test, 1 of the 2 implants in the femur was prepared. An incision was made to the lateral femur. Without putting pressure on the screws, the bone was reached with careful dissection. The torque test was applied at a speed of $1.0 \mathrm{~mm} / \mathrm{min}$ of the pull-out apparatus of the device (Lutron TQ-8800, Taiwan) (Fig. 3). The highest torque value obtained at the moment of breaking was recorded on a graphic and the digital screen as Newton units $(\mathrm{N})$.

Histomorphometry allows evalution of the biological fixation of the implant at the microscopic level and provides quantitative data. With this method, metallic implants are cut in situ and implant- bone interface is evaluated while the damage during removing is prevented. This assesment method prolonged use with mechanical test in orthopedic implants, oral surgery

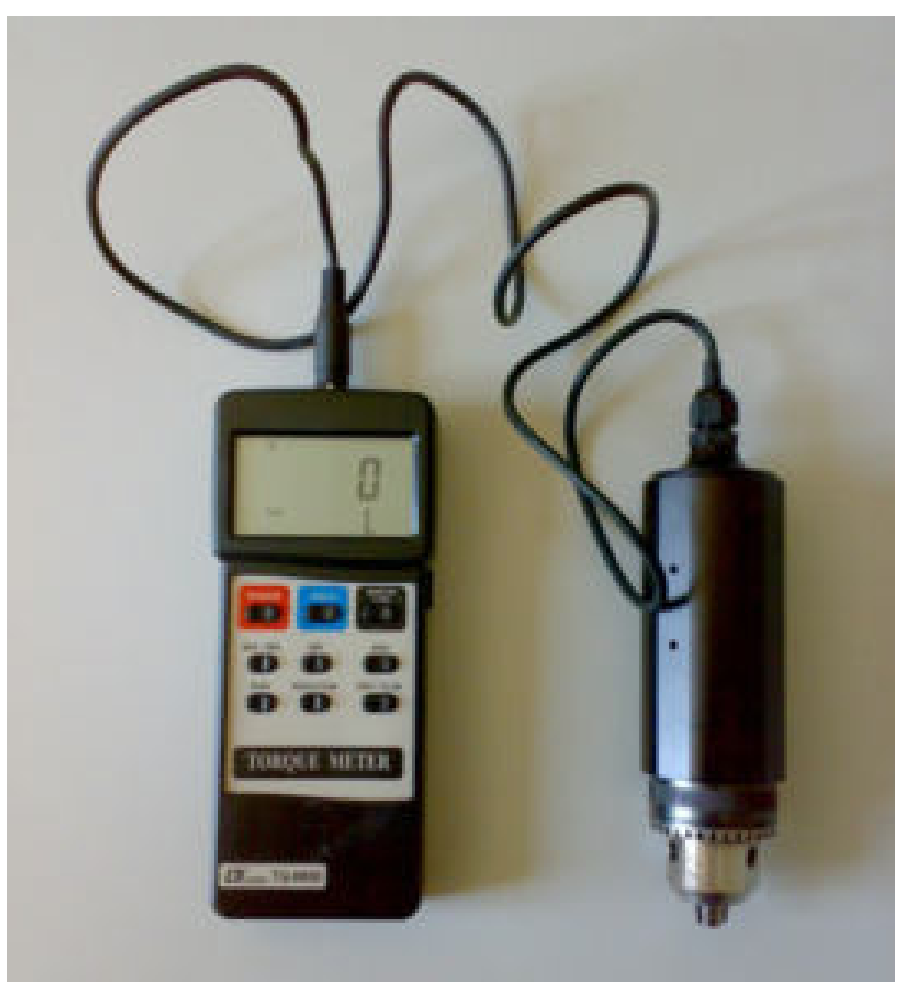

Fig. 2. Photograph shows the torque-meter device 


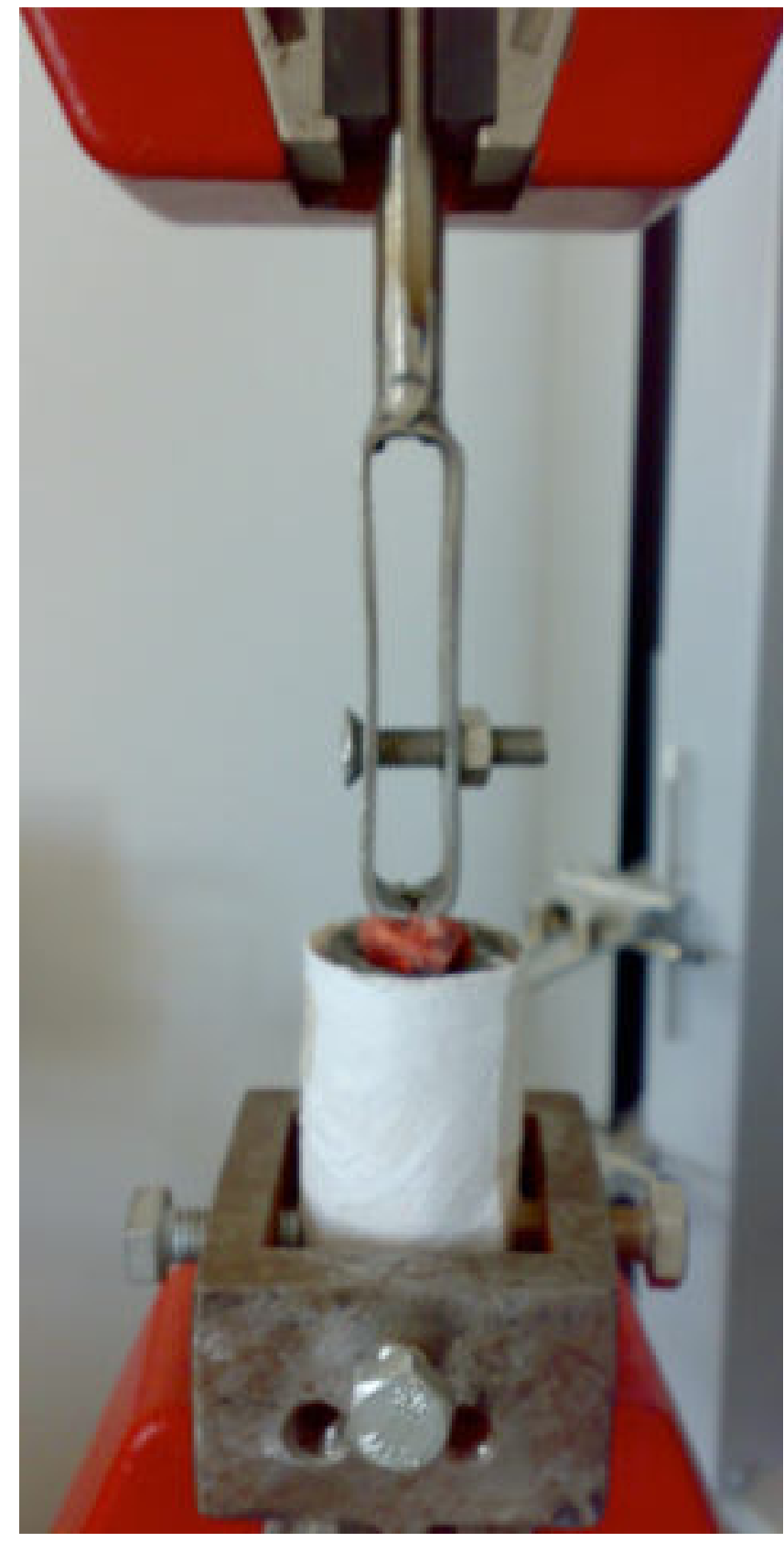

Fig. 3. Photograph shows the pull-out apparatus.

and maxillofacial surgery, and in the evalution of osteointegration so determine long- term implant survival.

In 3 rabbits of each group (6 rabbits, 12 screws), the distal femoral metaphysis was resected as the screws together with the surrounding bone for histomorphometric evaluation. Sections were taken and stained without decalcification of the samples and histomorphometric evaluation was made for comparison between the groups at the microscopic level. Digital photographs of the prepared sections were taken under a light microscope at $\times 4$ magnification and the images were recorded on the computer (Olympus DP 70, Tokyo, Japan). Measurements on the recorded images were calculated as Bone Implant Contact (BIC)percentages using a semi-automatic image analysis program (Image J 1.43u, Wayne Rasband, National Institute of Health, USA). The BIC calculation was made using the following formula:

\section{Bone-Implant contact length \\ BIC $=$ Surrounding length of the whole implant 100}

\section{Statistical Analysis}

For statistical analysis, SPSS 13.0 (Windows v 13.0, SPSS Inc, Chicago, IL, USA) statistical software program was used. Pull-out torque, removal torque and BIC values were compared between the groups with and without antibiotics using the Mann Whitney U-test. Correlations of these values for the groups were examined with Pearson correlation analysis. Data were expressed as a mean, median, and standard deviation. A value of $p<0.05$ was accepted as statistically significant. This experimental study was performed with the least number of animals because of ethical principals and animal rights. The biomechanical and histomorphometric evaluation was performed with a similar number of subjects in the literature. Power analysis was calculated according to the experimental animal study of Moojen et al. [15] and 20 rabbits were included in the study.

\section{RESULTS}

In Group 2 (not antibiotic-coated), 1 rabbit died due to diarrhea and was replaced by the addition of a new rabbit that had been operated on. No other problems were encountered throughout the experiment.

In the pull-out test, the values of the screws in Group 2 (not antibiotic-coated) were higher than those of the screws in Group 1 (antibiotic-coated) [290.90N (Newton) (238.50-330.60), 195.41N (129.20-281.50); $p=0.009)$. In the removal torque test, the values of the screws in Group 1 (antibiotic-coated) were higher than those in Group 2 (not antibiotic-coated) $[10.07 \mathrm{~N} / \mathrm{cm}$ (6.3-11.7), 7.17N/cm (4.8-10.2); $p=0.017]$ (Table 1). No positive correlation was determined between the 
Table 1. Bone implant contact percentages, pull-out test values and removal torque test values

\begin{tabular}{lccc}
\hline & $\begin{array}{c}\text { Antibiotic coated group } \\
\text { median (min, max) }\end{array}$ & $\begin{array}{c}\text { Control group } \\
\text { median (min, max) }\end{array}$ & $p$ value \\
\hline Bone Implant Contact $(\mathbf{\%})$ & $48.11(36.90,79.10)$ & $57.66(47.80,73.50)$ & $\mathbf{0 . 1 3 2}$ \\
Removal Torque $\left(\mathbf{N} / \mathbf{c m}^{\mathbf{1}}\right)$ & $10.07(6.30,11.70)$ & $7.17(4.80,10.20)$ & $\mathbf{0 . 0 1 7}$ \\
Pull-out $\left(\mathbf{N}^{\mathbf{2}}\right)$ & $195.41(129.20,281.50)$ & $290.90(238.50,330.60)$ & $\mathbf{0 . 0 0 9}$ \\
\hline
\end{tabular}

${ }^{1}$ Newton/centimeter, ${ }^{2}$ Newton

pull-out test and the removal torque test $(\mathrm{r}=0.88)$. From the histomorphometric results, osteointegration was determined in both groups. Following the 4weeks healing period, new bone formation was seen on the implant-bone interface under polarised light microscope imaging with toluidin blue staining. In Group 1, mean BIC was calculated as $48.11 \%(36.9-79.1)$ and in Group 2, mean BIC was $57.66 \%$ (47.8-73.5). In the general evaluation of the groups, although the BIC value of Group 1 was lower than that of Group 2, the difference was not determined as statistically significant $(p=0.132)$.

\section{DISCUSSION}

In this study, the effect on bone-implant osteointegration of a direct coating of teicoplanin on titanium screws was investigated and the results showed that teicoplanin coating did not interfere with osteointegration biomechanically or histomorphometrically. To the best of our knowledge, there are no other studies in the literature which have researched the effect of teicoplanin coating on osteointegration.

In an experimental study, the application of tobramycin to PA coated titanium foam on cylindrical titanium rods appeared to have a beneficial effect on implant fixationin a rabbit Staphylococcus aureus infection model histomorphometrically, which would result in improved longterm implant survival. Although the difference was not statistically significant $(21 \pm$ $3 \%$ for PA-tobra, $16 \pm 3 \%$ for PA), it was reported that the effect of tobramycin and periapatite coating on osteointegration was relatively better. In the same study, it was reported that there was a high local antibiotic effect of tobramycin and periapatite coating as infection prophylaxis in uncemented implants and it was concluded that it could be useful for biological fixation which is important for the longterm survival of the prosthesis. However, due to the suppression of infections with local tobramycin coated implants of a group of rabbits in that study, the effect of the antibiotic coating on osteointegration could not be evaluated independently [15]. Gentamycin hydroxyapatite and gentamycin RGD (arginine-glycine-aspartate) coatings of wires expressed antibiotics for up to 2 days and new bone formation were not inhibitedas well [5]. In another experimental rabbit study in which antibiotic soaked microparticles were used, it was shown that the coating with these microparticles was both protectives against infection and did not affect osteointegration [9]. In our study, we used titanium screws in metaphyseal area of rabbit femurs which we think screws imitate the cementless femoral stems and acetabular cups because they actually have primary stabilization through the metaphyseal region and as if they lock in with increasing diameters. In addition, we did not use an infection model in the current study and eliminate the disadvantage of suppression of infection so that we could evaluate the effect of antibiotic coating just on osteointegration contrary to the abovementioned study.

In the current study, to evaluate the connecting force of the implant to the bone, the pull-out and removal torque tests were applied as biomechanical test methods. Using the removal torque test, three-dimensional evaluation is made possible of the interface between the bone and the implant and is accepted as an important test in the evaluation of osteointegration $[16,17]$. This method focuses primarily on the properties of the conflicting forces related to the interface [13]. In cases where the removal torque test could be standardized, it has been reported to be a reliable method by many researchers [13]. There are a limited number of studies on the use of temporary implants such as mini implants in clinical studies as it is 
an invasive test [14]. By creating shearing force in the implant-bone interface, this test aims to measure the breaking resistance of this connection. Forced rotation against the direction in which the implant has been tightened inside the bone results in increased tension in the interface and a break occurring at the point at which it exceeds maximum resistance strength of the implant-bone connection, which causes movement of the implant [18]. In studies where the removal torque test has been applied, force scales such as digital torquemeter and digital dynamometer have been used $[13,18]$.

One of the findings of the current study was that different results were obtained from the groups in the pull-out and removal torque tests. The removal torque test is known to be used in the measurement of biomechanical properties of the implant-bone interface, whereas the pull-out test shows the biomechanical properties of the bone surrounding the implant [19]. In the pull-out test evaluating the bone around the implant, the nonantibiotic coating group values were higher but these values were not as valuable as the removal torque test, as 4-week-time was early in terms of completely mature bone formation around the implant. Long periods of up to 24 weeks are required for the complete connection between the bone and the implant material and for bone remodeling to be fully completed [20]. Therefore, as can be understood from this, the results of the removal torque test are more useful in the evaluation of osteointegration. The lower values of the antibiotic coating group in the pull-out test can be considered to be because of the relatively minor percentage values in the bone-implant contact of the screws of this group in the histomorphometric analysis.

In our study, the connection between the bone and the implant was examined with the histomorphometric method. Histomorphometry presents data which provide the possibility of quantitative evaluation of the biological fixation of theimplant at the microscopic level [5]. With this method, metallic implants are evaluated in situ thus avoiding cutting and damage to the implant-bone interface when removing the implant. Therefore, osteointegration is an evaluation method which has been used for a long time together with mechanical tests in the determination of implant survival in dental surgery and maxillofacial surgery and in the evaluation of orthopedic implants $[12,19$, 21].

One might argue that the mini-screws that we used in our study did not reflect all the orthopedic devices. But, we put the screws in metaphyseal area of the long bone of rabbit femur and this site is the primary stabilizing site of cementless femoral stems and acetabular cups in terms of arthroplasty. We think that this site reflects osteointegration of the cementless prosthesis. A Kirschner wire coated with phage and linezolid was used in one study and authors made conclusions about arthroplasty. Gentamycin hydroxyapatite and gentamycin RGD (arginine-glycin-aspartate) coatings of wires had similar amounts of new bone formation regarding the proximal and distal metaphyseal area of rabbit tibia [5]. It is hard to simulate the cementless prosthesis in animals since a wire which has been implanted intramedullary had been used in animal studies and biomechanical loading of these wires which is crucial for new bone formation is not an appropriate method. Because of those reasons, we studied the metaphyseal region for osteointegration which is important for primary stability of cementless femoral stems and acetabular cups in joint replacement. Additionally, we used screws and we think that it also imitates the acetabular prosthesis since it also locks into the acetabulum. Besides, the screws naturally reflect the other orthopedic fixation devices. We also studied the effect of direct teicoplanin coverage on bone ingrowth. One advantage of our study is the histomorphometric analysis showing the bone-implant contact directly and numeric comparison could be done easily between groups. In the study of Moojen et al. [15],tobramycin periapatite coating on titanium implantshas been shown to have better osteointegration histomorphometrically.

Teicoplanin is a glycopeptide antibiotic and has a broad spectrum, including gram-positive aerobic and anaerobic bacteria as well as methicillin-resistant S. Aureus (MRSA) [22-26]. The observation that teicoplanin can penetrate into muscle and bone tissues has made its use parenterally common in bone and joint infections [27]. It is very effective against all staphylococci, streptococci, enterococci, and pneumococci. In the current study, we used the technique described by Darouiche et al. [12] for antibiotic coating. In this technique, there is a direct antibiotic 
coating. It has been shown to be effective in vitro and in vivo against Staphylococcus aureus infection in a rabbit model [11].

\section{CONCLUSION}

In conclusion, when it is considered that direct coating of titanium screws with teicoplanin did not inhibit osteointegration, which is important in the biological contact of prosthetic implants, this method can be recommended to be used especially in implants with expected long-term function. Further experimental and clinical studies are required on this subject.

\section{Acknowledgments}

No data from other published sources are used in this study. We thank all medical technicians of Experimental Animal Research Center of Uludag University Veterinary Faculty.

\section{Conflict of interest}

The author disclosed no conflict of interest during the preparation or publication of this manuscript.

\section{Financing}

The authors disclosed that they did not receive any grant during conduction or writing of this study.

\section{REFERENCES}

1. Bruellhoff K, Fiedler J, Möller M, Groll J, Brenner RE. Surface coating strategies to prevent biofilm formation on implant surfaces. Int J Artif Organs 2010;33:646-53.

2. Del Pozo L, Patel, R. Infection associated with prosthetic joints. N Engl J Med 2009;361:787-94.

3. Matthews P, Berendt A, McNally M, Byren I. Diagnosis and management of prosthetic joint infection. BMJ 2009;338: b1773. 4. ter Boo GJ, Grijpma DW, Moriarty TF, Richards RG, Eglin D. Antimicrobial delivery systems for local infection prophylaxis in orthopedic and trauma surgery. Biomaterials 2015;52:113-25. 5. Alt V, Bitschnau A, Böhner F, Heerich KE, Magesin E, Sewing A, et al. Effects of gentamicin and gentamicin-RGD coatings on bone ingrowth andbiocompatibility of cementless joint prostheses: an experimental study inrabbits. Acta Biomater 2011;7:1274-80.

6. Guyer RD, Abitbol JJ, Ohnmeiss DD, Yao C. Evaluating osseointegration into a deeply porous titanium scaffold: a biomechanical comparison with peek and allograft. Spine (Phila $\mathrm{Pa}$ 1976) 2016;41:E1146-50.
7. Lin X, Yang S, Lai K, Yang H, Webster TJ, Yang L. Orthopedic implant biomaterials with both osteogenic and anti-infection capacities and associated in vivo evaluation methods. Nanomedicine 2017; 13:123-42.

8. Eltorai AE, Haglin J, Perera S, Brea BA, Ruttiman R, Garcia DR, et al. Antimicrobial technology in orthopedic and spinal implants. World J Orthop 2016;7:361-9.

9. Ambrose CG, Clyburn TA, Mika J, Gogola GR, Kaplan HB, Wanger A, et al. Evaluation of antibiotic-impregnated microspheres for the prevention of implant-associated orthopedic infections. J Bone Joint Surg Am 2014;96:128-34.

10. Ordikhani F, Dehghani M, Simchi A. Antibiotic-loaded chitosanLaponite filmsfor local drug delivery by titanium implants: cell proliferation and drug releasestudies. J Mater Sci Mater Med 2015;26:269.

11. Aykut S, Öztürk A, Özkan Y, Yanik K, Ilman AA, Özdemir R. Evaluation and comparison of the antimicrobial efficacy of teicoplanin and clindamycincoated titanium implants. J Bone Joint Surg Br 2010;92-B:159-63.

12. Darouiche RO, Mansouri MD, Zakarevicz D, Alsharif A, Landon GC. In vivo efficacy of antimicrobial-coated devices. J Bone Joint Surg Am 2007;89:792-7.

13. Johansson CB, Han CH, Wennerberg A, Albrektsson T. A quantitative comparison of machined commercially pure titanium and titanium-aluminum-vanadium implants in rabbit bone. Int $\mathrm{J}$ Oral Maxillofac Implants 1998;13:315-21.

14. Lages FS, Douglas-de Oliveira DW, Costa FO. Relationship between implant stability measurements obtained by insertion torque and resonance frequency analysis: a systematic review. Clin Implant Dent Relat Res 2018;20:26-33.

15. Moojen DJ, Vogely HC, Fleer A, Nikkels PG, Higham PA, Verbout AJ, et al. Prophylaxis of infection and effects on osseointegration using a tobramycin-periapatite coating on titanium implants an experimental study in the rabbit. J Orthop Res 2009;27:710-6.

16. Wennerberg A, Albrektsson T, Andersson B, Krol JJ. A histomorphometric and removal torque study of screw-shaped titanium implants with three different surface topographies. Clin Oral Implants Res 1995;6:24-30.

17. Ercan E, Candirli C, Arin T, Kara L, Uysal C. The effect of Er,Cr:YSGG laser irradiation on titanium discs with microtextured surface morphology. Lasers Med Sci 2015;30:11-5.

18. Favero LG, Pisoni A, Paganelli C. Removal torque of osseointegrated mini-implants: an in vivo evaluation. Eur J Orthod 2007;29:443-8.

19. Ogle OE. Implant surface material, design, and osseointegration. Dent Clin North Am 2015;59:505-20.

20. Ni S, Li X, Yang P, Ni S, Hong F, Webster TJ. Enhanced apatite-forming ability and antibacterial activity of porous anodic alumina embedded with $\mathrm{CaO}-\mathrm{SiO} 2-\mathrm{Ag} 2 \mathrm{O}$ bioactive materials. Mater Sci Eng C Mater Biol Appl 2016;58:700-8.

21. Barfeie A, Wilson J, Rees J. Implant surface characteristics and their effect on osseointegration. Br Dent J 2015;218:E9.

22. Bryson DJ, Morris DL, Shivji FS, Rollins KR, Snape S, Ollivere BJ. Antibiotic prophylaxis in orthopedic surgery: difficult decisions in an era of evolving antibiotic resistance. Bone Joint J 2016;98-B(8):1014-9. 
23. Wright GD. Antibiotic adjuvants: rescuing antibiotics from resistance. Trends Microbiol 2016;24:862-71.

24. Chirca I, Marculescu C. Prevention of infection in orthopedic prosthetic surgery. Infect Dis Clin North Am 2017;31:253-63.

25. Wang T, Li N, Hu S, Xie J, Lei J, Wang Y, et al. Factors on trough teicoplanin levels, associations between levels, efficacy, and safety in patients with gram-positive infections. Int J Clin Pharmacol Ther 2015;53:356-62.
26. Göçer H, Önger ME, Kuyubaşı N, Çıraklı A, Kır MÇ. The effect of teicoplanin on fracture healing: an experimental study. Eklem Hastalik Cerrahisi 2016;27:16-21.

27. Mihatovic I, Golubovic V, Becker J, Schwarz F. Immunohistochemical analysis of staged guided bone regeneration and osseointegration of titanium implants using a polyethylene glycol membrane. Clin Oral Investig 2014;18:429-35. 